\title{
Exploring Geomorphic Processes and Martian Gale Crater Topography on Mars using CTX and HiRISE Express Image Dataset
}

1. Graphical Abstract

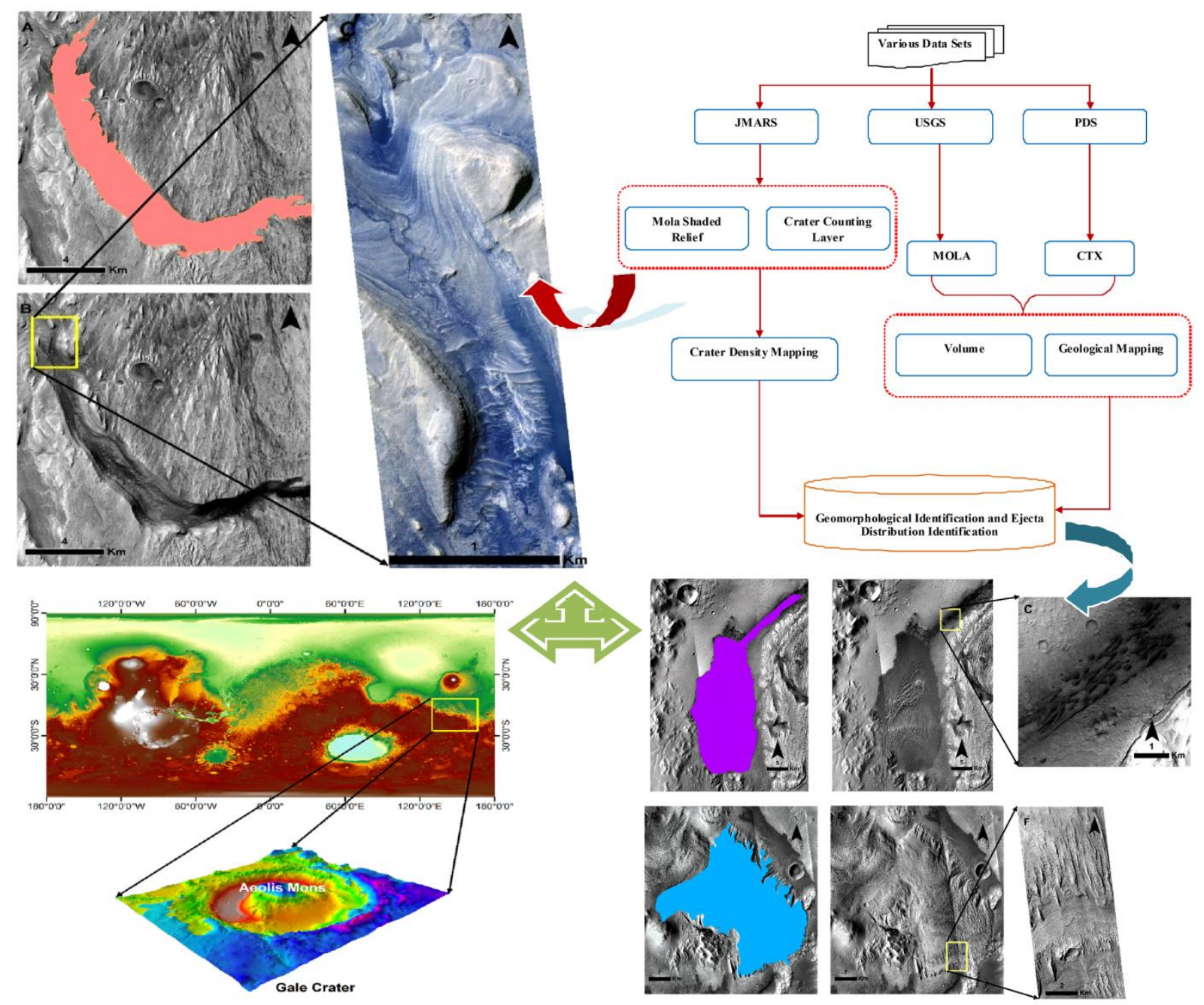

Fig.1. Graphical abstract showing mapping and identification of geomorphological features 


\section{Calibration algorithm of CTX Images}

CTX images with 6 meters spatial resolution obtained from the MRO satellite were used for mapping the Martian geomorphological features (Fig. 2). Radiometric calibration of raw CTX image can be done with image manipulation using direct sets of calculations. The process of radiometric calibration not only enhanced the image quality for interpretation but also reduced the digital number (DN) values errors.

\subsection{Decompanding}

All CTX data within the instrument electronics are companded into 8-bit (0-255 DN) data files from their original 12-bit (04095 DN) format usinga square-root-like compression lookup table (LUT) by using the CCD gain and read noise during preflight camera testing developed by the CTX team.

\subsection{Bias and Dark Current Subtraction and Even/Odd striping correction}

During each CTX exposure both current signals i.e. bias and dark accumulate simultaneously. Although, small levels between the A and B channels of the CCD array can still differ slightly. In order to subtract this background signal level, a very simple process can be used. For this, the first step is to average DN values calculated separately in the odd (channel A) and even (channel B) masked-off reference pixels (pixels 1-38 and 5039-5056) and at second step, the calculated average values were subtracted from their corresponding odd and even unmasked pixels (pixels 39-5038). For additional cosmetic improvement of CTX images another small correction may be applied that attempts to explicitly remove any systematic detector gain effects that possibly exist between the channels A and B of the CTX detector.

Subtracted and flat fielded CTX row was scaled to radiance using the CTX response coefficient derived during pre-flight calibration using the equation:

$$
I F=\left(\text { pix }_{\text {val }} / \exp \right) /\left(\text { resp }_{\text {coef }} * \text { sol_spect_rad }\right)
$$

Where,pix $x_{\text {val }}$ is the pixel value being evaluated in DN, exp is the exposure time in msec,resp $p_{\text {coef }}$ is the CTX response coefficient in $(\mathrm{DN} / \mathrm{msec}) /\left(\mathrm{W} / \mathrm{m}^{2} / \mu \mathrm{m} / \mathrm{sr}\right)$, andsol_spect_rad is the solar spectral radiance for CTX in $\left(\mathrm{W} / \mathrm{m}^{2} / \mu \mathrm{m} / \mathrm{sr}\right)$.

\subsection{Calibration algorithm of HiRISE image}

The radiometric correction process improves the interpretation and quality of images and reduces the errors in digital number (DN) values. The radiometric correction of the image was determined as: 


$$
Z=([H-B 0 * L)] / L) * A 0 * G * I *(1.5 / A U) 2
$$

Where, $\mathrm{H}=$ Output of hiclean (offset and dark current corrected image); $\mathrm{B}_{0}=$ Intra-channel dark current correction (BIN and TDI dependent); $A_{0}=$ Intra-channel gain correction (BIN and TDI dependent); $\mathrm{G}=$ Global gain correction; $\mathrm{L}=$ Observation line time; $\mathrm{I}=\mathrm{I} / \mathrm{F}$ conversion factor at Sun-Target distance of $1.5 \mathrm{AU} ; \mathrm{AU}=$ Distance between Mars and Sun during observation time; $\mathrm{Z}=$ Corrected image by radiometrically in $\mathrm{I} / \mathrm{F}$ units.

Intensity flux (intensity/flux, $\mathrm{i} / \mathrm{f}=1$ ) was calculated by converting RDR image DN integer value to flux for getting the normal view of ideal Lambertian reflector to the surface. Intensity/flux values were obtained using the following equation:

$\mathrm{I} / \mathrm{F}=(\mathrm{DN} *$ Scaling factor $)+$ Offset
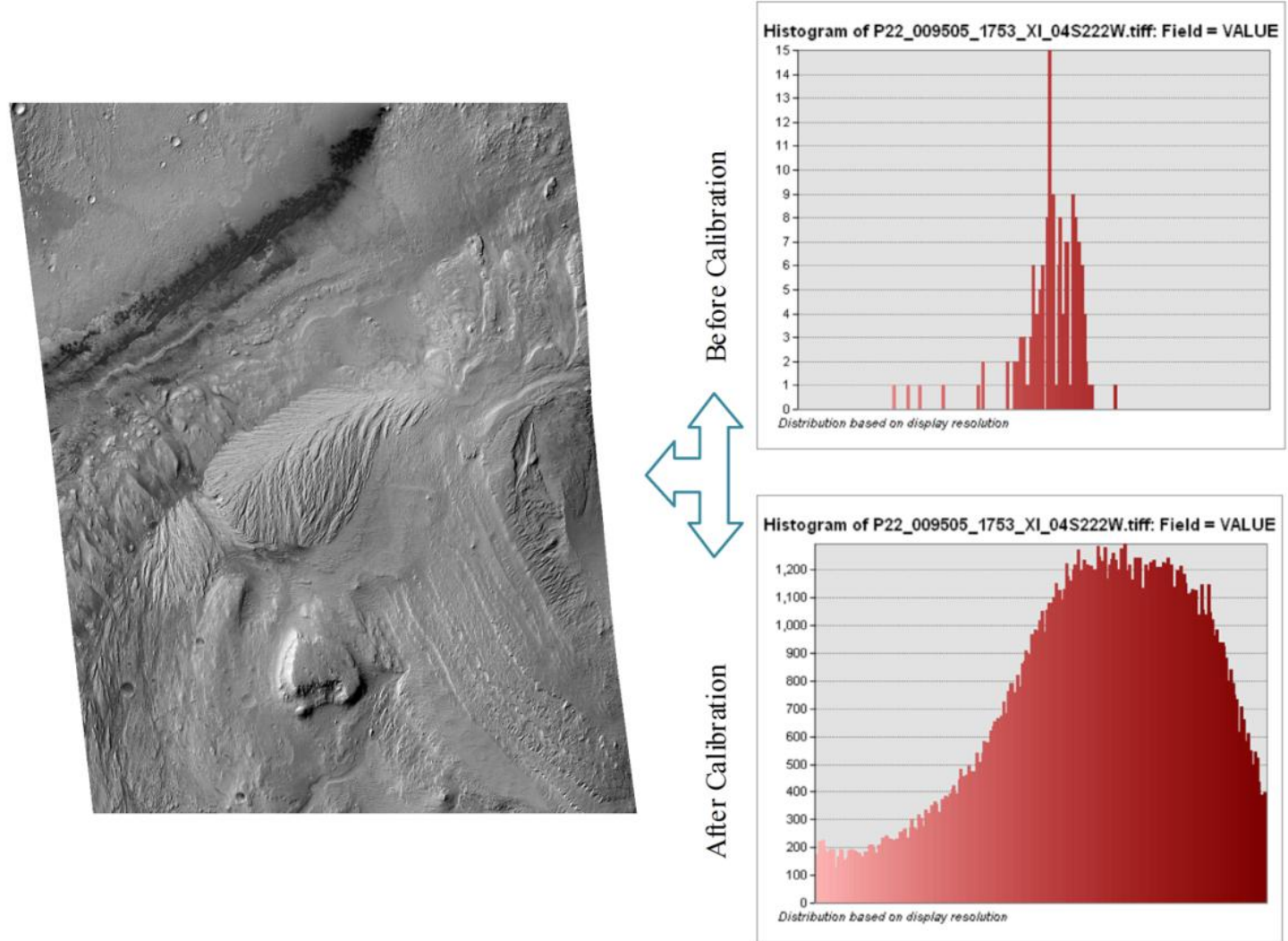

Fig. 2. Processed HiRISE image and calibration algorithm histogram 


\section{Statistical Relationship among SLE, DLE, MLE and RLE Morphology}

Gale Crater $\left(5.3^{\circ} \mathrm{S}, 222.3^{\circ} \mathrm{W}\right)$ with $170 \mathrm{~km}$ in diameter liesbetween the southern cratered highlands and Elysium Planitia lowlands in north-eastern part of the Aeolis quadrangle.On mars, the morphologies of impact crater are quite different from the craters on other planets, which are formed by ballistic sedimentation. The renewed Martian topography comprises layers or ejecta morphology. These ejecta morphologies can be categorized into single(SLE), double(DLE), multiple(MLE) and radial layers (RLE).

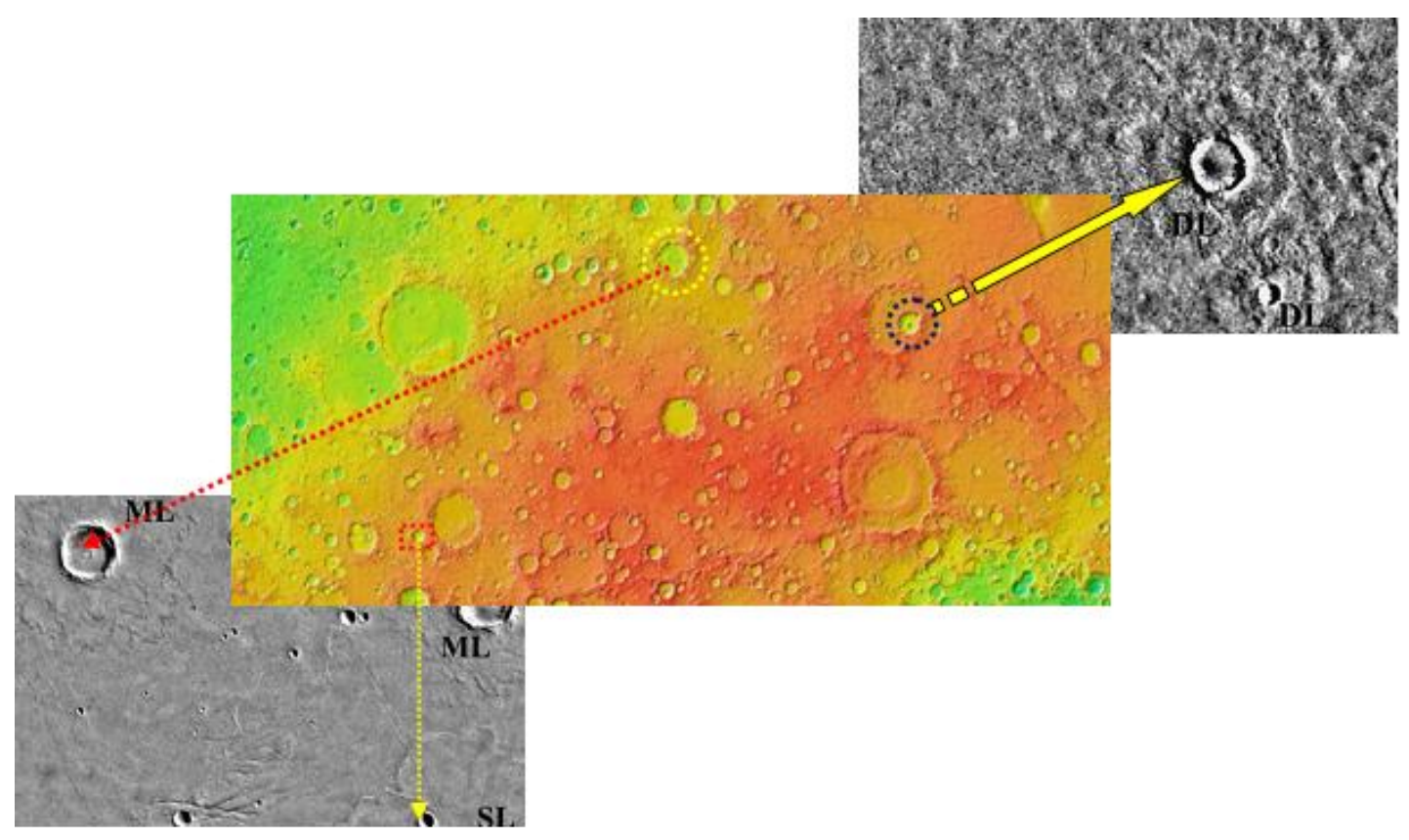

Fig. 3. SLE, MLE and DLE ejecta morphologies

The current study finds that symmetrical central pit has highest correlation between crater diameter and pit diameter (Fig. 4). The single layer ejecta was found concentrated between equatorial latitude while double, multiple and radial layer were observed at middle latitudes. These varied topographic features are very much significant in idealizing the surface conditions on Martian topography.The percentage of ejecta was calculated for every 10 latitude on the Martial surface (Fig. 11). 

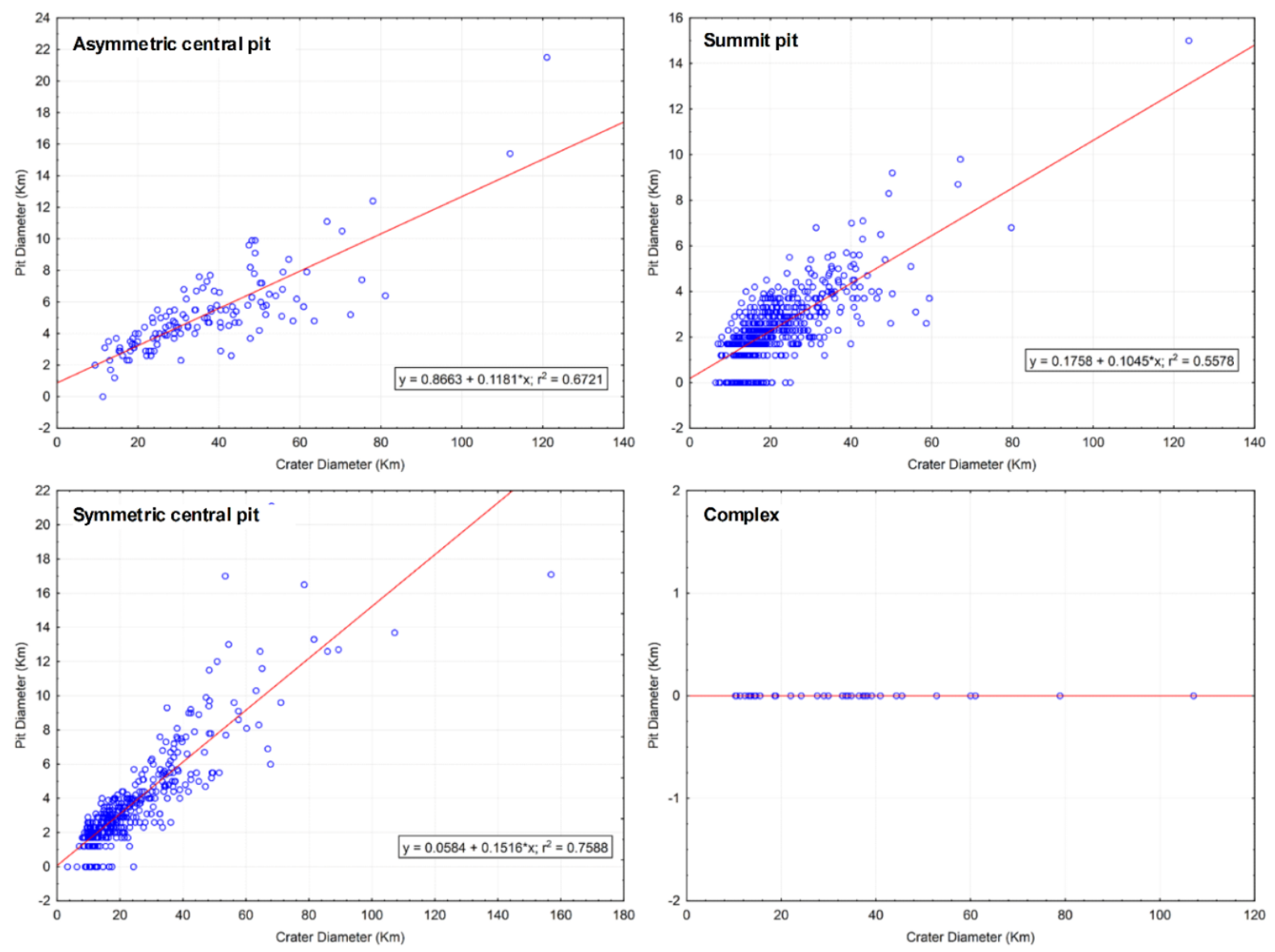

Fig.4. Relationship between crater diameter of pit diameter of SLE, DLE, MLE, and RLE

It means that if the diameter of crater is increases then the pit diameter also increases but it is not true in cases of complex craters. On the other hand, the relationship between crater diameter and pit diameter for the radial layer ejecta is strongest $\left(r^{2}=0.7947\right)$ than the single, double and multiple layer ejecta but the numbers of crater for the radial layer ejecta is less than other craters (Fig. 5). 

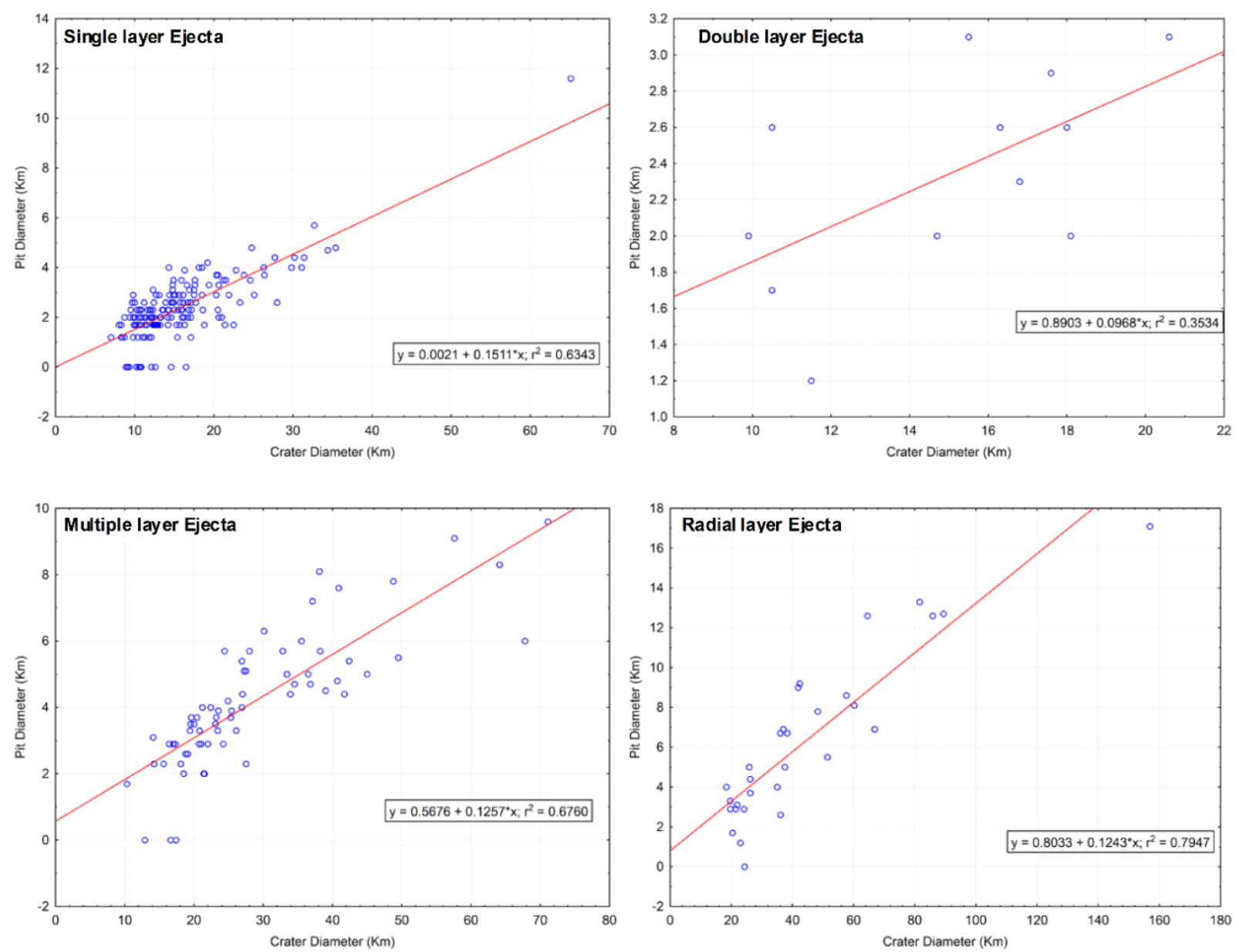

Fig. 5. Symmetrical interior morphology for different individual ejecta morphology

The number of craters showing summit pit is very less in comparison with symmetrical interior morphology. It indicated that relationship between ejecta morphology and interior morphology is insignificance (Fig. 6).In case of asymmetrical pit interior morphology, the correlation between crater diameter and pit diameter is highest fordouble layerejecta $\left(\mathrm{r}^{2}=0.97\right)$ among different individual ejecta asshown in Fig. 7. 

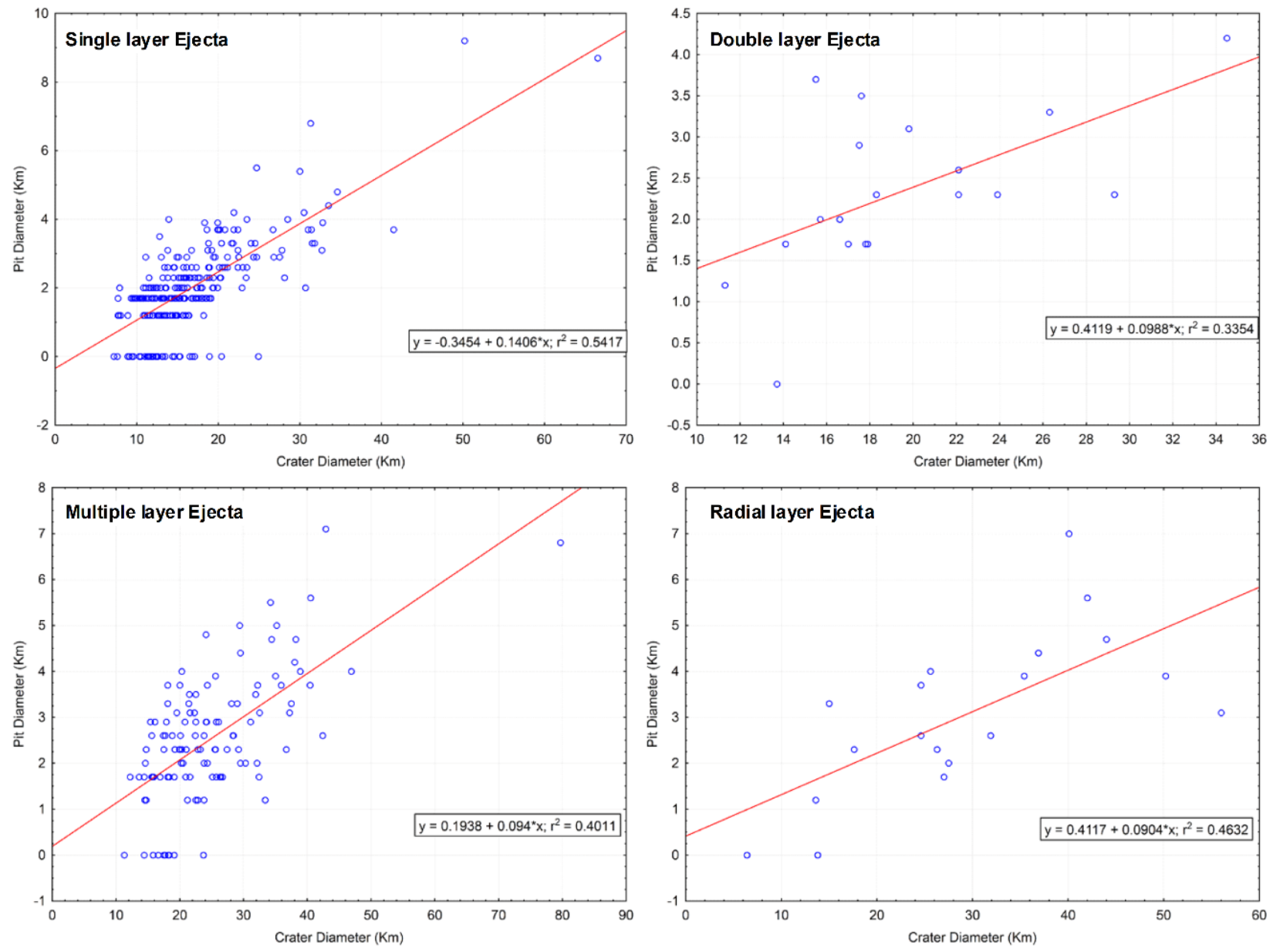

Fig. 6. Summit pit interior morphology for different individual ejecta morphology 

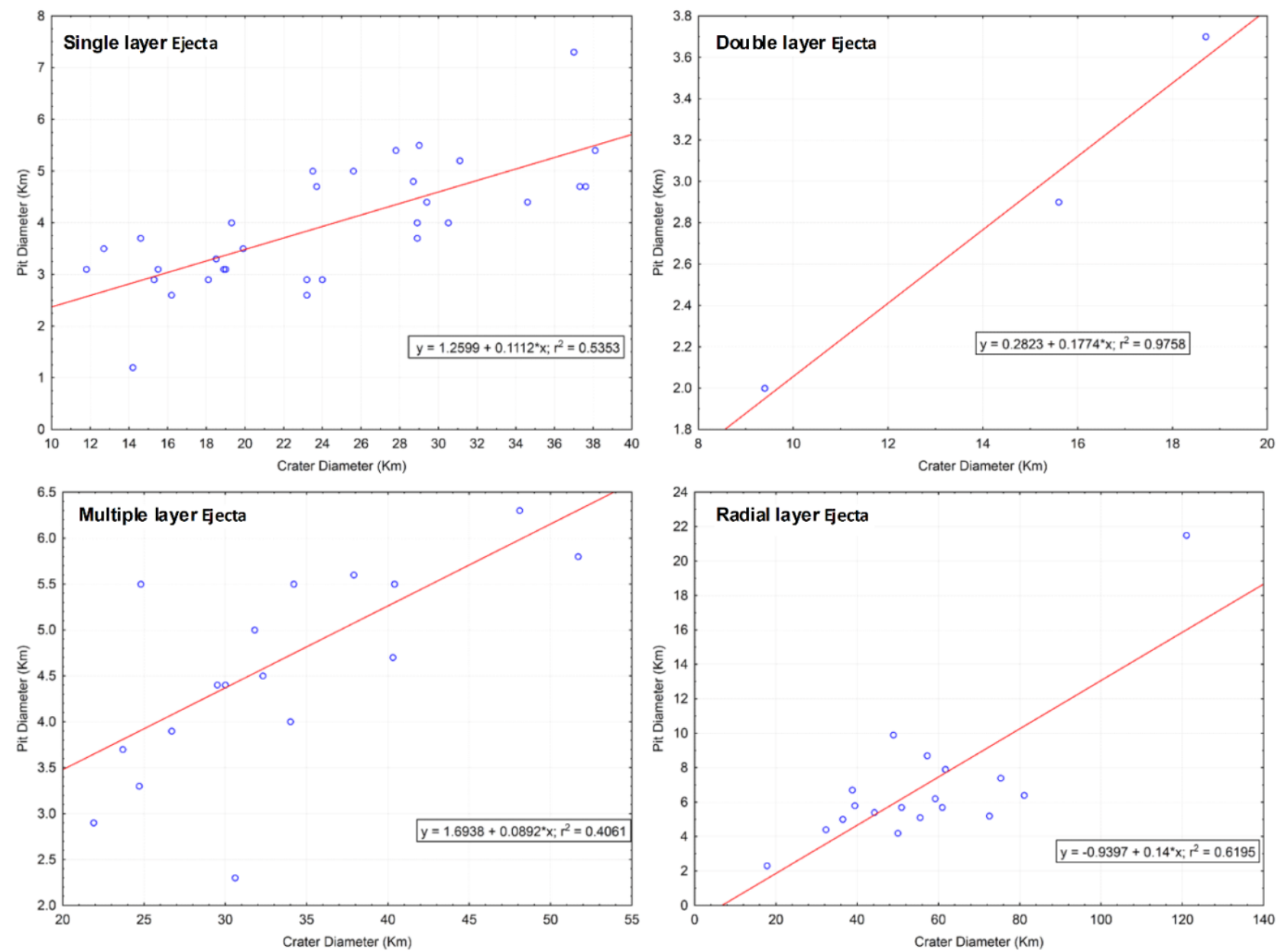

Fig. 7. Asymmetrical pit interior morphology for different individual ejecta morphologyat different latitudes 